\title{
A study of learning and retroactive inhibition under unilateral cortical depression'
}

GORDON SPIES

LAKE FOREST COLLEGE

With unilateral cortical depression induced by $\mathrm{KCl}$, seven rats learned a complex maze-reversal as easily as they had the original maze with both cortices functional. Recall of the original maze, with a hemisphere not subjected to interference, was as good after six days as it was for controls after $4 \mathrm{hr}$.

Spreading depression induced by $\mathbf{K C l}$ applied to the cortex of one hemisphere of the rat does not spread to the other (Bures, Buresova, \& Zaharova, 1958), and a simple CAR established in the functional hemisphere does not transfer to the other (Bures \& Buresova, 1960; Tapp, 1962; Travis \& Spark, 1963).

This study was undertaken to determine if, with unilateral depression, the rat can learn more complex tasks than previously demonstrated, and to ascertain if the $\mathrm{KCl}$ technique might provide a means for resolving the issue of retroactive Inhibition as the sole cause of forgetting versus decay of the memory trace as a contributory factor.

Subjects

Twenty male albino Spraque-Dawley rats, 100 days old, were randomly assigned to the experimental (E) and control (C) groups.

\section{Apparatus}

The apparatus consisted of six $\mathrm{T}$-sections (stem $12 \times 4-1 / 2 \times 4-1 / 2$ in., arms 7-1/2 $\times 4-1 / 2 \times 4-1 / 2$ in. each) with lockable hinged door at the end of each arm, plus a start box and a gaal box (12 x 4-1/2 $x$ 4-1/2 in, each), all with Plexiglas lids. The T-sections could be joined together in any sequence to form a six-choice-point maze.

Ss were housed in cages, 9-1/2 $\times 7 \times 7$ in., with smooth galvanized-iron sides and backs, Plexiglas fronts, and fine wire mesh floors to reduce the possibility of cannulae being pulled out.

\section{Procedures}

In the manner described by Tapp (1962) cannulae (1 $\mathrm{cm}$ long, ID $2 \mathrm{~mm}$ ) were implanted to make contact with the dura $2 \mathrm{~mm}$ each side of the midsagittal suture and $2 \mathrm{~mm}$ posterior to the coronal suture. The cannulae were filled with cotton soaked in isotonic saline, and a press-fit plug closed each tube. The isotonic-soaked cotton was replaced daily.

All rats were placed on a 23-hr. food deprivation schedule 10 days before surgery of the experimental Ss (ESs) and maintained on this schedule throughout the experiment, except that ESs were fed ad libitum for the first two days of a five-day post-operative period.

\section{Training-Experimental Subjects (ESs)}

Stage 1. ES was placed in the start box of Maze A and allowed to make its way to the goal box. The door at the end of each blind alley was locked and the door at the end of each correct alley closed behind ES when traversed. ES received five $45 \mathrm{mg}$ food pellets as reinforcement and was immediately started on the next trial. Six trials per day were given till ES reached a criterion of two error-free runs in succession $(p<.01)$. An error was counted if ES placed both forepaws over the line marking the beginning of a blind alley. Thirty min. after each daily session, food was made avallable for $1 \mathrm{hr}$.

Stage 2. The day following ES's achievement of criterion in Maze $A$, the $T$-sections were scrambled and the order of turns reversed to form Maze $B$. The isotonic-soaked cotton in one cannula was removed and replaced with $20 \% \mathrm{KCl}$ (no cotton), and the tube replugged. Thirty min. later ES's six trials in Maze $B$ began. On completion of the training session, the $\mathrm{KCl}$ was removed and replaced by isotonic-soaked cotton, and ES returned to its home cage. Thirty min. later food was avallable to it for $1 \mathrm{hr}$. This regimen was followed daily till Maze B was learned to the same criterion as in Stage 1.

Stage 3. The day after ES achieved criterion in Stage 2, the moze was returned to the A sequence and the same procedure as in Stage 2 followed, except that the opposite cortex of ES was depressed while the cortex depressed in Stage 2 remained functional.

Stage 4. The day following achievement of criterion in Stage 3, the cortex of ES depressed in Stage 2 was again depressed, the cortex depressed in Stage 3 remaining functional. The same procedure as heretofore was followed, with Maze A again in use.

Training-Control Subjects (CSs)

Stage l. CSs were trained in Maze A under the same conditions and to the same criterion as ESs in Stage 1.

Stage 2. Four hr. after reaching criterion, CSs were tested for retention in Maze A.

Stage 3. The day after completion of Stage 2, six trials per day in Maze $B$ were commenced and continued until criterion reached.

\section{Results and Discussion}

In a pilot study a $25 \% \mathrm{KCl}$ solution induced such severe contralateral clonus that rats were unable to negotiate the maze. The $20 \%$ solution finally used also induced clonus, but not sufficiently to impair locomotion materially. The pilot study also showed 


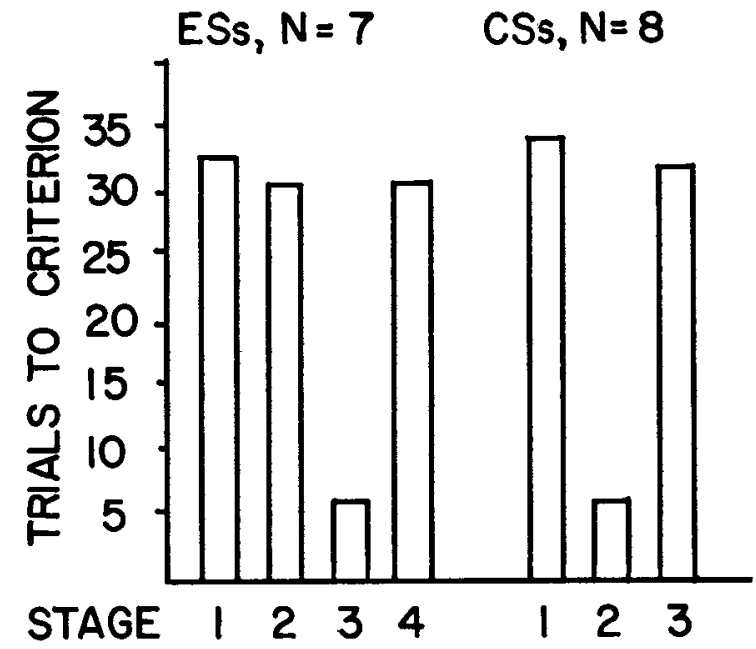

Fig. 1. Average number of trials taken by $\mathrm{Ss}$ to reach criterion at their respective procedural stages.

that the $20 \%$ solution could not be left in a cannula overnight. Three of four animals on which this was tried were dead the following morning; the fourth was inert, but recovered. No rat with unilateral depression showed any evidence of reduction of appetite.

During the study two ESs and two CSs developed respiratory infections and were discarded, while one ES pulled out its cannulae.

Figure 1 shows average number of trials taken by ESs and CSs to reach criterion at their respective procedural stages. For ESs the differences between Stages 1 and 2,1 and 4 , and 2 and 4 were not significant, while differences between Stages 1 and 3 , 2 and 3 , and 3 and 4 were highly significant $(p<.01)$. For CSs the difference between Stages 1 and 3 was not significant, while differences between Stages 1 and 2 and 2 and 3 were highly significant $(p<.01)$.

Comparing results for ESs at Stages 1 and 2 and, similarly, 2 and 4 , it is apparent that ESs under unilateral depression learned maze-reversal as easily as they had the original maze with both cortices functional. Similarly, no impairment of maze-reversal learning is indicated when these results are compared with CSs' results at Stages 1 and 3 . Per contra, Travis \& Spark (1963) found that the learning of a simple CAR under unilateral depression was inferior to learning with both cortices functional. The overlearning trials to which CSs were subjected at Stage 2 may be a confounding factor here, there being little agreement as to whether over-learning aids or impairs reversal-learning in the rat (Sperling, 1965).

A comparison of results for ESs at Stage 3 with those for CSs at Stage 2 shows that, for the former, retention in the hemisphere not subjected to interference in the experimental situation is as good six days after reaching criterion as it is for CSs after $4 \mathrm{hr}$.

In conclusion, this study has demonstrated that the rat with unilateral cortical depression learns a complex task as well as it does with both hemispheres functional. While the study also demonstrates the severe effect of retroactive inhibition on recall, the inability of the rat to tolerate continuous application of $\mathrm{KCl}$ to the cortex, over a period of time necessary to permit the possibility of ruling out decay of the memory trace as a factor in forgetting, seems to preclude the use of the technique in the resolution of this issue.

\section{References}

Bures, J., \& Buresova, O. The use of Leao's spreading depression in the study of interhemispheric transfer of memory traces. $J$. comp. physiol. Psychol., 1960, 53, 558-563.

Bures, J., Buresova, O., \& Zaharova, A. Conditioned reflexes and Leao's spreading cortical depression. J. comp. physiol. Psychol., $1958,51,263-268$.

Sperling, Sally E. Reversal learning and resistance to extinction: A review of the rat literature. Psychol. Bull., 1965, 63, 281-297.

Tapp, J. T. Reversible cortical depression and avoidance behavior in the rat. J. comp. physiol. Psychol., 1962, 55, 306-308.

Travis, R. P. Jr., \& Sparks, D. L. The influence of unilateral and bilateral spreading depression during learning upon subsequent relearning. J. comp. physiol. Psychol., 1963, 56, 56-59.

Note

1. The author gratefully acknowledges support for this investigation from Lake Forest College. 